\title{
OPEN Investigation of the thermal tolerance of silicon-based lateral spin valves
}

\author{
N. Yamashita ${ }^{1}$, S. Lee ${ }^{1}$, R. Ohshima ${ }^{1}$, E. Shigematsu ${ }^{1}$, H. Koike' ${ }^{2}$, Y. Suzuki ${ }^{3}$, S. Miwa ${ }^{3,4}$, \\ M. Goto ${ }^{3}$, Y. Ando ${ }^{1}$ \& M. Shiraishi ${ }^{1}$
}

Improvement in the thermal tolerance of Si-based spin devices is realized by employing thermally stable nonmagnetic (NM) electrodes. For Au/Ta/Al electrodes, intermixing between $\mathrm{Al}$ atoms and $\mathrm{Au}$ atoms occurs at approximately $300^{\circ} \mathrm{C}$, resulting in the formation of a Au/Si interface. The Au-Si liquid phase is formed and diffuses mainly along an in-plane direction between the Si and AIN capping layers, eventually breaking the MgO layer of the ferromagnetic (FM) metal/MgO electrodes, which is located $7 \mu \mathrm{m}$ away from the NM electrodes. By changing the layer structure of the NM electrode from $\mathrm{Au} / \mathrm{Ta} / \mathrm{Al}$ to $\mathrm{Au} / \mathrm{Ta}$, the thermal tolerance is clearly enhanced. Clear spin transport signals are obtained even after annealing at $400^{\circ} \mathrm{C}$. To investigate the effects of $\mathrm{Mg}$ insertion in $\mathrm{FM}$ electrodes on thermal tolerance, we also compare the thermal tolerance among $\mathrm{Fe} / \mathrm{Co} / \mathrm{MgO}, \mathrm{Fe} / \mathrm{Co} / \mathrm{Mg} / \mathrm{MgO}$ and $\mathrm{Fe} / \mathrm{Co} / \mathrm{MgO} /$ $\mathrm{Mg}$ contacts. Although a highly efficient spin injection has been reported by insertion of a thin $\mathrm{Mg}$ layer below or above the MgO layer, these thermal tolerances decrease obviously.

Silicon ( $\mathrm{Si}$ ) is a suitable material for semiconductor-based spintronic devices because of its long spin lifetime ${ }^{1,2}$, excellent controllability of spin current by gate voltage ${ }^{3,4}$, and availability of mature infrastructures of Si-based electronic devices. Significant advances, including room-temperature demonstrations of Si-based spin devices such as a spin metal-oxide semiconductor field-effect transistor ${ }^{4-9}$ and a magneto logic gate ${ }^{10,11}$, have been achieved to date by using a magnesium oxide $(\mathrm{MgO})$ tunnel barrier. Recent studies have clarified the spin scattering mechanism in Si based on Elliott-Yafet mechanisms ${ }^{2,12,13}$, and optical phonon emissions suppress the spin diffusion length under high electric fields with applied gate voltage ${ }^{3}$. A remaining problem for the practical application of spin devices is a small signal amplitude in transistor and logic operations due to an insufficient spin accumulation voltage in Si. Thus, exploring a ferromagnetic contact enabling a large spin accumulation voltage in $\mathrm{Si}$ is a significant research issue, and half-metallic materials ${ }^{14-16}$ and the insertion of a thin magnesium $(\mathrm{Mg})$ layer ${ }^{9,17-20}$ in a ferromagnetic contact in Si spin devices have been extensively investigated. Among these methods, the insertion of a thin $\mathrm{Mg}$ layer above/below the $\mathrm{MgO}$ layer is a new approach to enhancing spin signals in spin devices. Indeed, the insertion of the $\mathrm{Mg}$ layer above the $\mathrm{MgO}$ layer allows for the enhancement of the spin polarization of $\mathrm{Fe}$ on $\mathrm{Mg} / \mathrm{MgO}^{9,17,18}$ due to suppression of the formation of a magnetic dead layer, and the insertion of $\mathrm{Mg}$ below the $\mathrm{MgO}$ layer provides a highly textured $\mathrm{MgO}$ tunnelling barrier on the Si channel, which enhances the magnitude of spin signals ${ }^{20}$.

Recently, we established and reported another way to increase spin signals in nondegenerate Si-based spin devices, which involves thermal annealing the Si spin devices at $300^{\circ} \mathrm{C}$, and a twofold increase in spin accumulation voltage was realized ${ }^{21}$. This enhancement was attributed to improved crystallinity in the $\mathrm{MgO}$ tunnelling barrier and better spin polarization. However, no spin accumulation signals were detected in that study after annealing above $300^{\circ} \mathrm{C}^{21}$, which presents a new problem for practical applications. The thermal tolerance of $\mathrm{Si}$ spin devices at least above $350^{\circ} \mathrm{C}$ is strongly desired to obtain compatibility with the infrastructures of Si-based electronic devices since the post fabrication process often employs thermal treatments above $300{ }^{\circ} \mathrm{C}^{22-24}$. Therefore, it is significant to clarify the degradation mechanism of Si spin devices by thermal annealing and to solve the problem of further progress in Si spintronics.

Here, we have investigated the thermal degradation mechanisms of a lateral spin valve (LSV) based on nondegenerate n-type Si by electric and crystallographic methods. It is revealed that the in-plane long-range diffusion of gold (Au) atoms in nonmagnetic (NM) electrodes occurs through the aluminium nitride (AlN)/

${ }^{1}$ Department of Electronic Science and Engineering, Kyoto University, Kyoto, Kyoto 615-8510, Japan. ${ }^{2}$ Advanced Products Development Center, TDK Corporation, Ichikawa, Chiba 272-8558, Japan. ${ }^{3}$ Graduate School of Engineering Science, Osaka University, Toyonaka, Osaka 560-8531, Japan. ${ }^{4}$ Institute for Solid State Physics, The University of Tokyo, Kashiwa, Chiba 277-8581, Japan. ${ }^{凶}$ email: yamashita.naoto.64r@st.kyoto-u.ac.jp 
a
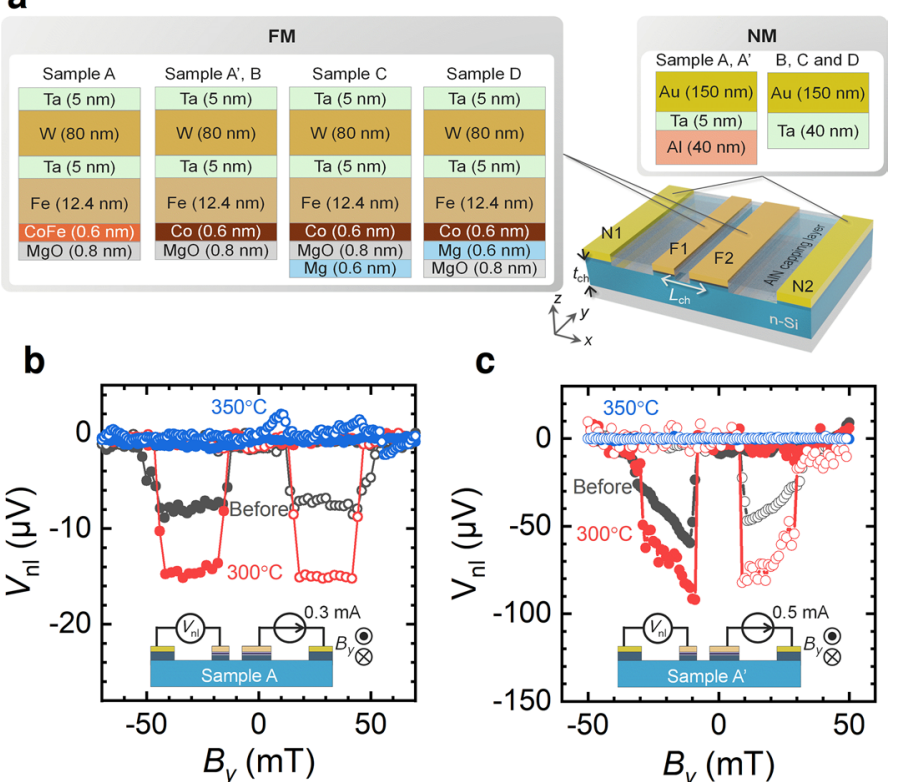

C
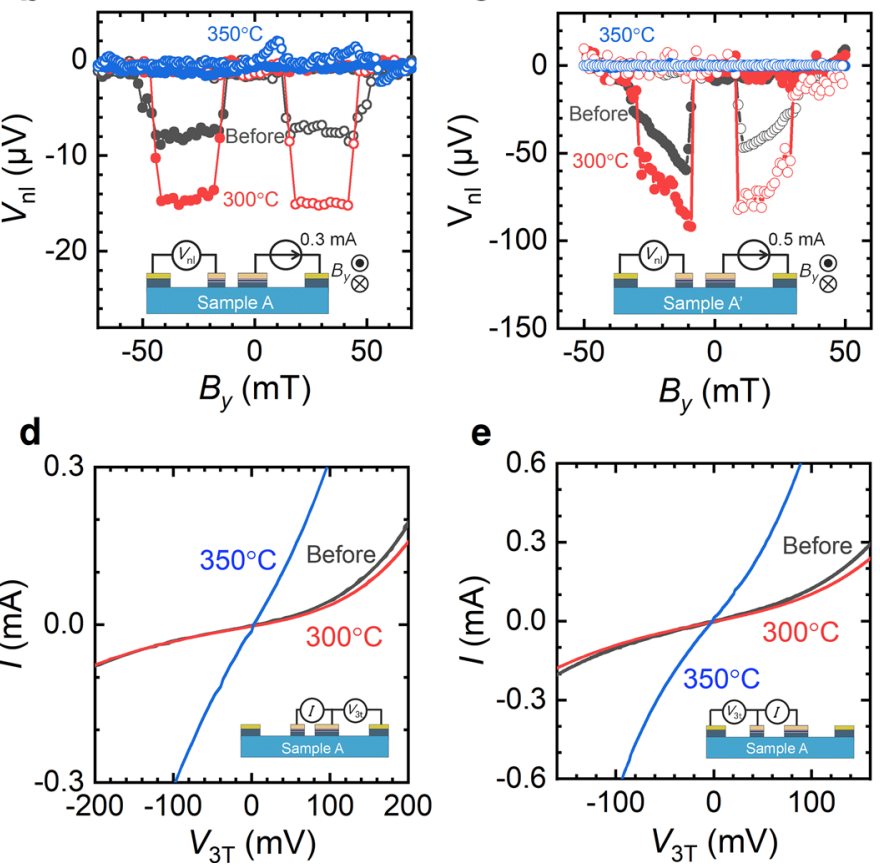

Figure 1. (a) A schematic of Si-based lateral spin valves. The channel width along $y$ direction was $21 \mu \mathrm{m}$. (b) Spin accumulation signals, i.e., nonlocal voltage $V_{\mathrm{nl}}$ as a function of magnetic flux density along the $y$ direction $B_{y}$, for sample A before annealing (black) and after annealing at $300^{\circ} \mathrm{C}$ (red) and $350{ }^{\circ} \mathrm{C}$ (blue), obtained by the NL4T method. The channel length $L_{\mathrm{ch}}$ was $2.0 \mu \mathrm{m}$, and the channel thickness $t$ was $80 \mathrm{~nm}$. The widths of F1 and F2 were 0.2 and $1.2 \mu \mathrm{m}$, respectively. (c) Spin accumulation signals of sample A' before annealing (black) and after annealing at $300{ }^{\circ} \mathrm{C}$ (red) and $350{ }^{\circ} \mathrm{C}$ (blue), obtained by the NL4T method. The channel length $L_{\mathrm{ch}}$ was $1.6 \mu \mathrm{m}$, and the channel thickness $t$ was $100 \mathrm{~nm}$. The widths of F1 and F2 were 0.5 and $2.0 \mu \mathrm{m}$, respectively. (d, e) $I-V$ characteristics of (d) F1 and (e) F2 before annealing (black) and after annealing at $300{ }^{\circ} \mathrm{C}$ (red) and $350^{\circ} \mathrm{C}$ (blue) for sample A.

$\mathrm{Si}$ interface. The Au atoms eventually reach a ferromagnetic (FM) electrode that is located $7 \mu \mathrm{m}$ away from the NM electrodes, resulting in the degradation of the MgO tunnelling barrier. To improve the thermal tolerance, the layer structure of the $\mathrm{NM}$ electrode is changed from $\mathrm{Au} / \mathrm{Ta} / \mathrm{Al}$ to $\mathrm{Au} / \mathrm{Ta}$. The modified device clearly exhibits spin signals even after annealing at $400{ }^{\circ} \mathrm{C}$. We also focus on the thermal tolerance of different structures in FM contacts without $\mathrm{Mg}$ insertion and $\mathrm{Mg}$ insertion below/above the $\mathrm{MgO}$ layer, as enhancement of the spin polarization has been reported by insertion of a thin $\mathrm{Mg}_{\text {layer }}{ }^{17,18,20}$. However, the thermal tolerance obviously decreases down to approximately $300{ }^{\circ} \mathrm{C}$ due to the existence of the $\mathrm{Mg}$ layer.

\section{Results and discussion}

Schematics of the device geometry and layer structure of the Si-based LSVs are shown in Fig. 1a. The devices consist of n-type Si, two FM electrodes (F1 and F2), two NM electrodes (N1 and N2) and an AlN capping layer on the Si channel. The details of sample preparation are described in the Methods section. The spin transport properties, such as the spin polarization and the spin diffusion length, were measured by nonlocal four-terminal $(\mathrm{NL} 4 \mathrm{~T})^{25}$ and local three-terminal (L3T) ${ }^{26}$ measurements at room temperature. Crystallographic analyses of the devices were carried out by means of cross-sectional transmission electron microscopy (TEM) with energy dispersive X-ray spectroscopy (EDS).

First, sample A was employed to investigate the thermal tolerance and degradation mechanism of the spin transport properties. The spin signals were measured by means of NL4T measurements, and the $I-V$ characteristics of F1 and F2 are measured at $300 \mathrm{~K}$. Spin accumulation signals, i.e., a nonlocal voltage $V_{\mathrm{nl}}$, as a function of $B_{y}$ before and after thermal annealing, are shown in Fig. 1b. A clear rectangular shape was measured, indicating 

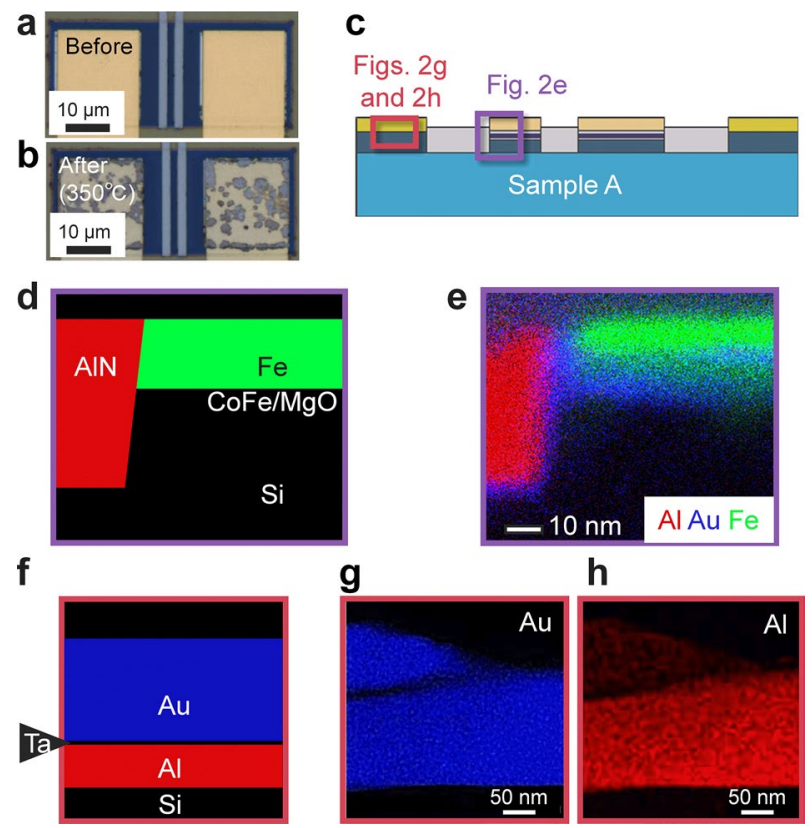

Figure 2. Microscopic pictures of sample A after annealing at $350^{\circ} \mathrm{C}$. (a, b) Optical microscopic image (a) before and (b) after annealing at $350^{\circ} \mathrm{C}$. (c) A schematic cross-section of sample A. The areas of EDS observations are roughly indicated by purple and red squares. (d) A designed structure and (e) an elemental mapping image near the FM electrode indicated by the purple rectangle in (c), where $\mathrm{Au}, \mathrm{Al}$ and $\mathrm{Fe}$ are shown in blue, red and green, respectively. (f) Designed structure and elemental mapping images of (g) Au and (h) Al near the NM electrode indicated by the red rectangle in (c).

successful spin transport. The magnitude of the spin accumulation signals was increased after annealing at $300{ }^{\circ} \mathrm{C}$, consistent with our previous report ${ }^{21}$. In contrast, the spin accumulation signal completely disappeared after annealing at $350^{\circ} \mathrm{C}$. These results were observed in both devices using $\mathrm{CoFe}(\mathrm{Fe} / \mathrm{CoFe} / \mathrm{MgO} / \mathrm{Si})$ and $\mathrm{Co}(\mathrm{Fe} / \mathrm{Co} /$ $\mathrm{MgO} / \mathrm{Si}$ ) as shown in Fig. 1c, which implies that the heat tolerance of the FM electrodes does not affect the results, i.e., that of the NM electrodes governs the tolerance. To investigate the electrical properties of FM1 and FM2, three terminal $I-V$ characteristics are measured. Figure $1 \mathrm{~d}$, e exhibit the three terminal $I-V$ characteristics of $\mathrm{F} 1$ and F2 before/after annealing. Whereas the $I-V$ characteristics did not change after annealing at $300{ }^{\circ} \mathrm{C}$, they decreased significantly after annealing at $350^{\circ} \mathrm{C}$, which was consistent with our previous study ${ }^{21}$. The reduction in the spin signal is attributable to the reduction in the interfacial resistance from $1.5 \times 10^{-8}$ to $1.6 \times 10^{-9} \Omega \mathrm{m}^{2}$ in $\mathrm{F} 1$ and from $1.5 \times 10^{-8}$ to $4.5 \times 10^{-9} \Omega \mathrm{m}^{2}$ in F2 due to the conductance mismatch ${ }^{21,27}$. The one-dimensional spin diffusion model (see methods) also revealed that the spin polarization decreases from approximately $2.8 \%$ to less than $1.3 \%$ after annealing at $350^{\circ} \mathrm{C}$.

Figure 2a, b show optical microscopy images of sample A before annealing and after annealing at $350{ }^{\circ} \mathrm{C}$. Although a smooth gold surface was confirmed at the NM electrodes before annealing, a patchy pattern in gold, grey and blue was apparent after annealing. In contrast, no significant changes were observed on the surface of the FM electrodes. To reveal the degradation mechanism in detail, crystallographic analyses using TEM and EDS were carried out. A cross-sectional elemental mapping images obtained by EDS are shown in Fig. 2e, g, h. We examined the cross-section of the FM electrodes indicated by the purple square in Fig. 2c. While Au was not used in FM electrodes (Fig. 2d), Au atoms, shown in blue, migrate from the NM electrode and were found around the FM electrodes (Fig. 2e). These electrodes were separated $7 \mu \mathrm{m}$ away from each other. Then, we examined an $\mathrm{NM}$ electrode consisting of an $\mathrm{Au} / \mathrm{Ta} / \mathrm{Al}$ trilayer (Fig. 2f) using the cross-sectional elemental mappings shown in Fig. $2 \mathrm{~g}$, h, revealing that $\mathrm{Au}$ atoms diffused in the Al layer and reach the Al/Si interface. TEM observation was carried out to investigate the crystallographic properties of the NM/Si interface and the Si channel to trace the path of Au diffusion. We observed the areas indicated in Fig. 3a. Whereas intermixing of Si channels and Au atoms was recognized beneath the NM electrodes, as shown in Fig. 3b, the intermixing area was limited only beneath the NM electrode. Figure 3c, d show atomic-resolution TEM images and the Fourier transformation obtains a part of the [110] surface of the Si channel ca. $0.4 \mu \mathrm{m}$ away from the NM electrodes. The Fourier transformation shows hexagonal spot patterns, which is evident in the clear periodicity of the diamond structure, indicating no significant structural damage to the Si channel. On the contrary, the Au diffusing area along the AlN/Si interface shows a halo feature (Fig. 3e), indicating a significant degradation of the crystal structure. Since the Au-Si system exhibits the eutectic alloys with a considerably low solubility limit of Au atoms in the Si phase, a segregation of $\mathrm{Au}$ atoms is expected if the Si channel dominantly contributes the Au diffusion. Because no Au particles were confirmed near the bottom side of the Si layer, the Si channel is not a probable diffusion path for the Au atoms.

We then clarify the possible mechanism of $\mathrm{Au}$ atom diffusion by annealing. The $\mathrm{Al}$ and Si binary systems exhibit eutectic alloys, but the temperature of the eutectic point is above $570{ }^{\circ} \mathrm{C}$, indicating that the Al-Si liquid 
a

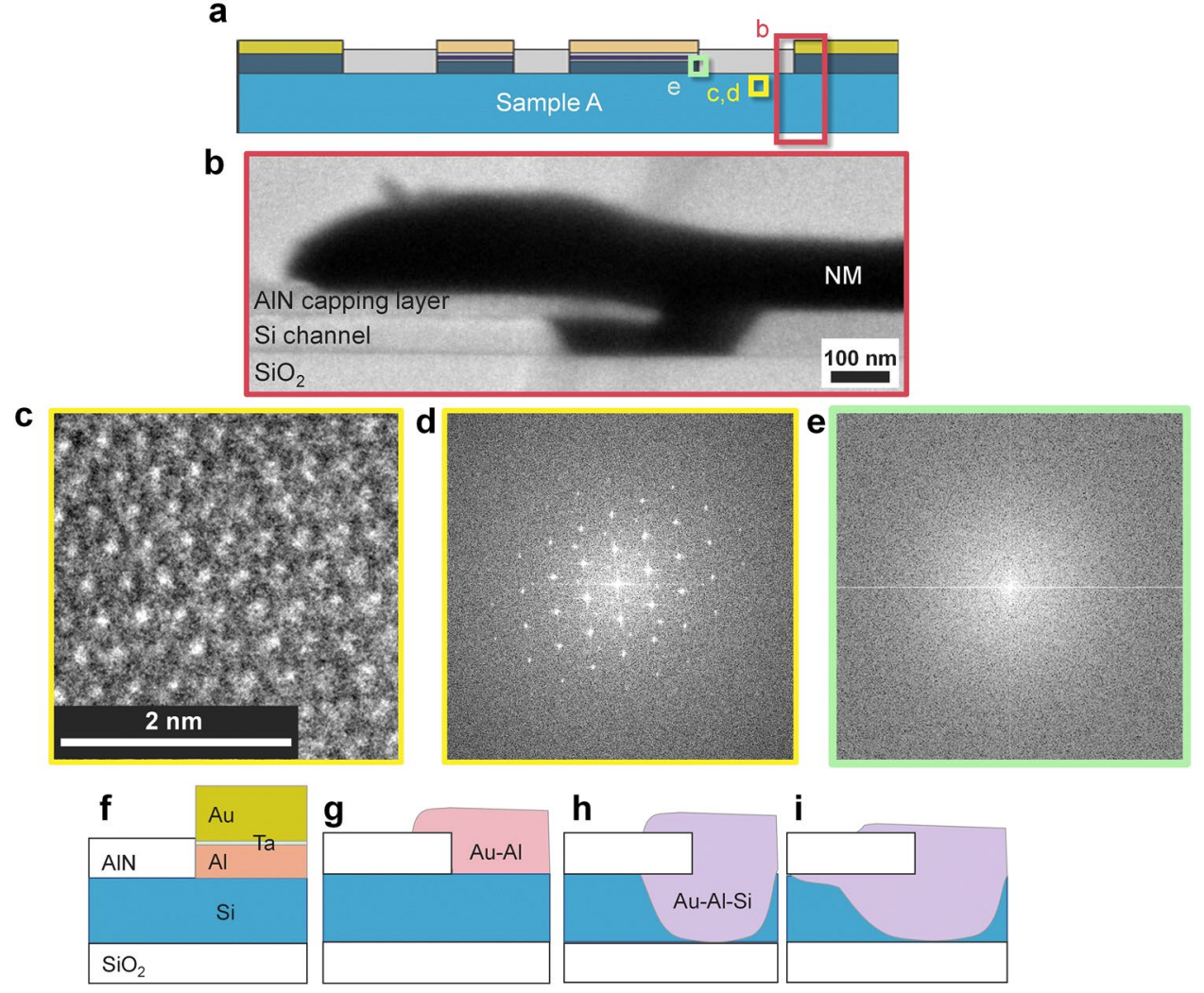

Figure 3. Crystallographic analyses of the Si channel after annealing and a possible degradation mechanism by annealing. (a) A schematic cross-section of sample A. The areas of TEM observations are roughly indicated by red and yellow squares. (b) A cross-sectional TEM image of the NM electrode obtained at the red square shown in (a). (c) An atomic-resolution TEM image obtained at the Si channel indicated by the yellow rectangle in (a) and (d) the Fourier transformation of the area. (e) A Fourier transformation of the area of Au diffusing along AlN/Si interface. (f)-(i) Schematic images of the possible mechanism of diffusion of the Au atoms during annealing. (f) the fabricated structure. (g) Au-Al intermetallics were formed, and Au reached Si. (h) The intermetal of Au-Al-Si was formed, including the liquid phase. (i) The liquid phase diffused through the interface of AlN/Si.

phase is not formed at the $\mathrm{Al} / \mathrm{Si}$ interface during the annealing process. Similarly, $\mathrm{Ta}-\mathrm{Si}$, Ta-Al and $\mathrm{Au}-\mathrm{Al}$ systems are expected to maintain their solid states below $400{ }^{\circ} \mathrm{C}$. In contrast, the temperature of the eutectic point of the $\mathrm{Au}-\mathrm{Si}$ system is approximately $350^{\circ} \mathrm{C}$. Considering the above situations, a possible mechanism of Au atom diffusion is as follows: The designed structure is shown in Fig. 3f. First, the intermixing of $\mathrm{Al}$ and Au atoms takes place below $300^{\circ} \mathrm{C}$, as schematically shown in Fig. $3 \mathrm{~g}$, which is confirmed by EDS (Fig. $2 \mathrm{~g}, \mathrm{~h}$ ) despite the 5 -nm Ta layer. The interdiffusion length $l$ of $\mathrm{Au}$ and $\mathrm{Al}$ atoms, estimated from $l=5.56 \times 10^{-10} t \mathrm{~m}$ at $350{ }^{\circ} \mathrm{C}^{28}$, is $2 \mu \mathrm{m}$ after $t=1 \mathrm{~h}$. Therefore, Au atoms easily penetrate the Al layer, which is only $40 \mathrm{~nm}$ in thickness, and finally reach the $\mathrm{Si}$ channel. Since the melting point of $\mathrm{Au}_{8} \mathrm{Si}_{2}$ is approximately $350{ }^{\circ} \mathrm{C}, \mathrm{Au}-\mathrm{Si}$ and/or $\mathrm{Au}-\mathrm{Al}-\mathrm{Si}$ liquid phases are formed at the $\mathrm{Au}-\mathrm{Si}$ interface (Fig. $3 \mathrm{~h}$ ). Because $\mathrm{Au}_{8} \mathrm{Si}_{2}$ is the eutectic point, additional $\mathrm{Au}$ and $\mathrm{Si}$ atoms rapidly increase the melting point, so most of the Si channel remains without reaction. The Au-Si and/ or Au-Al-Si liquid phases diffuse mainly along the AlN/Si interface (Fig. 3i) due to the strong tensile $\operatorname{strain}^{29}$, and the interface is more susceptible to destruction than the other areas. Finally, Au reaches the FM electrodes and invades the $\mathrm{MgO}$ tunnelling barrier. To confirm the repeatability, elemental mapping images of other parts at the AlN/Si interface of sample A were also obtained around the NM and FM electrodes as shown in Fig. 4. $\mathrm{Au}$ was detected at the AlN/Si interface even between the FM 1 and 2, i.e., a part of the spin transport channel as indicated by the arrows in Fig. 4b, c. Therefore, not only FM contacts but also the spin transport channel was damaged by the Au atoms.

To confirm the thermal tolerance of FM electrodes, a control experiment was performed using sample B. To circumvent the interdiffusion of $\mathrm{Al}$ and $\mathrm{Au}$, we excluded $\mathrm{Al}$ from the $\mathrm{NM}$ electrodes and employed a thick Ta layer: $\mathrm{Au}(150 \mathrm{~nm}) / \mathrm{Ta}(40 \mathrm{~nm})$. Ta is often used as a barrier metal for $\mathrm{Si}$ against conductive metals such as $\mathrm{Cu}$ because the Ta layer is maintained even after annealing at $630^{\circ} \mathrm{C}$, which is high enough for any thermal treatments applied to electronic devices ${ }^{30}$. Thermal annealing at $300{ }^{\circ} \mathrm{C}, 350{ }^{\circ} \mathrm{C}$, and $400{ }^{\circ} \mathrm{C}$ was carried out. The optical microscope image of sample B after annealing at $400^{\circ} \mathrm{C}$ showed no salient changes on the surface of the $\mathrm{Au} /$ Ta electrode (see Fig. 5a, b), which was in contrast to the $\mathrm{Au} / \mathrm{Ta} / \mathrm{Al}$ structure in sample A. Cross-sectional elemental mapping images (see Fig. $5 \mathrm{c}$ indicating the examined parts) showed no signal from Au atoms detected at the $\mathrm{AlN} / \mathrm{Si}$ interface or around $\mathrm{FM}$ even after annealing at $400^{\circ} \mathrm{C}$ (see Fig. 5d). Furthermore, the NM electrode 
a

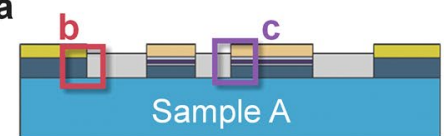

b

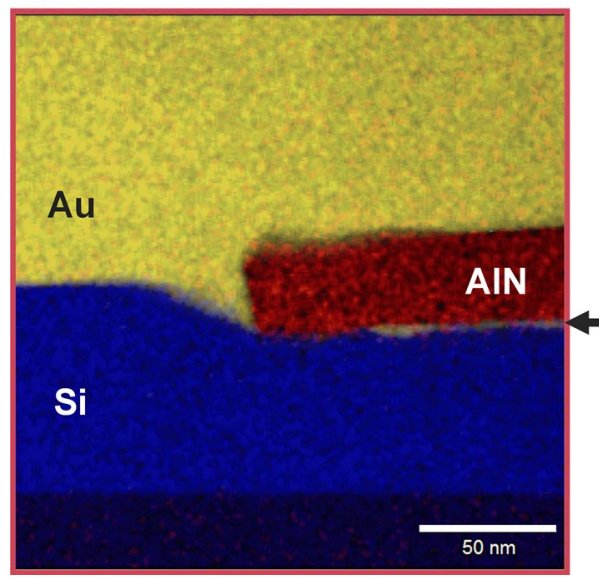

C

Figure 4. Elemental mapping images of other parts of sample A. (a) A schematic cross-section of sample A. The examined areas are indicated by the red $(\mathbf{b})$ and the purple (c) rectangles. (b) The overlay of the elemental mapping around N1. The blue, yellow, and red area indicate $\mathrm{Si}, \mathrm{Au}$, and N near the NM electrode. Au was detected from the AlN/Si interface, which is indicated by the arrow. (c) The overlay of the elemental mapping around F2. The blue, yellow, red, and green area indicate $\mathrm{Si}, \mathrm{Au}, \mathrm{N}$, and $\mathrm{W}$ near the FM electrode. Au was detected from the AlN/Si interface, which is indicated by the arrow.
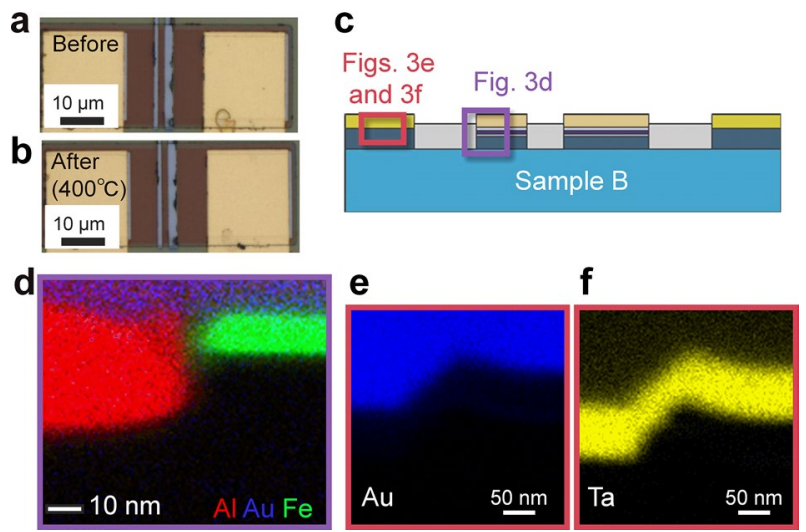

Figure 5. Microscopic pictures of sample $B$ after annealing at $400^{\circ} \mathrm{C}$. (a, b) Optical microscopic image (a) before and (b) after annealing at $400^{\circ} \mathrm{C}$. (c) A schematic cross-section of sample B. The areas of EDS observations are roughly indicated by purple and red squares. (d) Elemental mapping image near the FM electrode indicated by the purple rectangle in (c), where $\mathrm{Al}, \mathrm{Au}$ and $\mathrm{Fe}$ are shown in red, blue and green, respectively. (e, f) Elemental mapping images of (e) Au and (f) Ta near the NM electrode indicated by the red rectangle in (c).

clearly maintains the Ta/Au bilayer structure (see Fig. 5e, f). These results are obviously different from those of sample A, which directly supports our hypothesis of the thermal degradation of a Si-based LSV.

To confirm the thermal tolerance of the LSV, NL4T measurements were carried out. Clear hysteresis features were obtained for all conditions, i.e., before annealing and after annealing at 300,350 and $400{ }^{\circ} \mathrm{C}$, as shown in Fig. 6a. The magnitude of the spin accumulation signal before annealing was $9 \mu \mathrm{V}$, and the signal was enhanced to $18 \mu \mathrm{V}$ after annealing at $300^{\circ} \mathrm{C}$, similar to that of sample $\mathrm{A}$. In contrast to sample $\mathrm{A}$, the spin signal did not decrease after annealing at $350^{\circ} \mathrm{C}$. Furthermore, clear spin accumulation signals were also obtained even after annealing at $400^{\circ} \mathrm{C}$. To corroborate the tolerance of the spin diffusion length, Hanle signals were also measured in the NL4T method for sample B after annealing at 300 and $350{ }^{\circ} \mathrm{C}$, as shown in Fig. $6 \mathrm{~b}$. To eliminate the spurious effects, the difference in $V_{\mathrm{nl}}$ between the parallel and antiparallel magnetic configurations, $V_{\mathrm{nl}}^{\mathrm{P}}-V_{\mathrm{nl}}^{\mathrm{AP}}$, was 
a

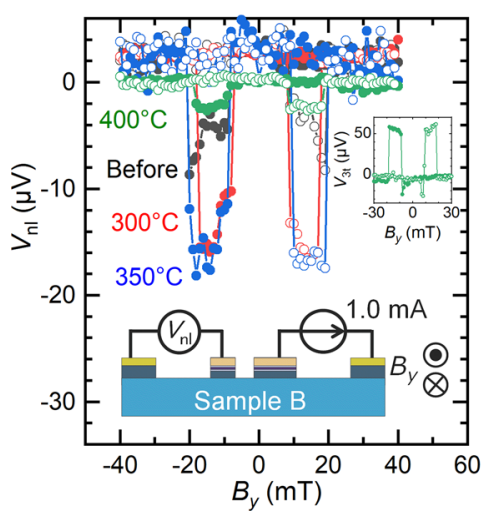

b

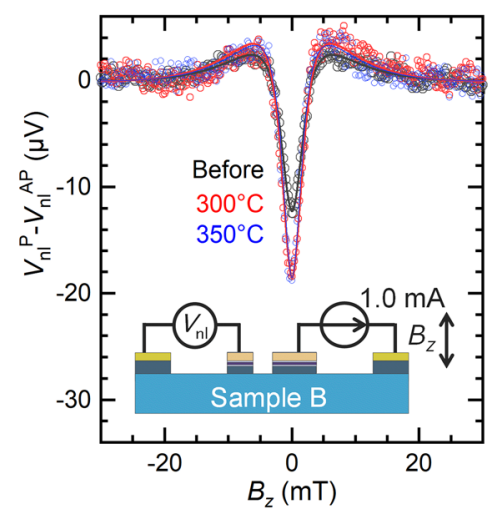

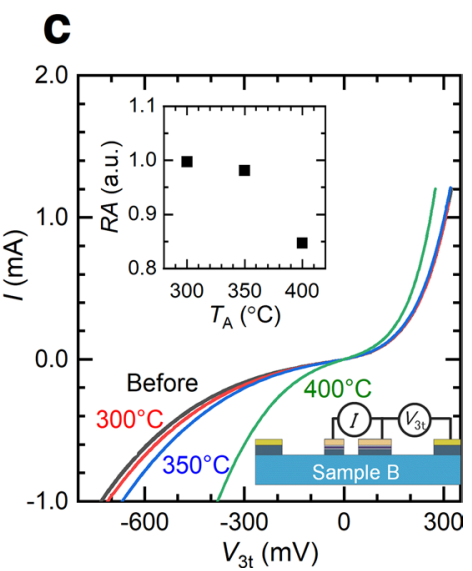

Figure 6. Results of the control experiments with sample B, which has NM electrodes made of Au/Ta. $L_{\mathrm{ch}}=2.75 \mu \mathrm{m}, t=100 \mathrm{~nm}$ for sample B. The widths of F1 and F2 were 0.5 and $2.0 \mu \mathrm{m}$, respectively. (a) The spin accumulation signals of sample B measured by the NL4T method. Black, red, blue and green show the measured $V_{\text {nl }}$ before annealing after annealing at $300^{\circ} \mathrm{C}, 350^{\circ} \mathrm{C}$ and $400{ }^{\circ} \mathrm{C}$, respectively. The inset shows the result of the L3T measurement after annealing at $400^{\circ} \mathrm{C}$. (b) Results of the Hanle measurements of sample B. Black, red and blue show the measured $V_{\mathrm{nl}}$ before annealing after annealing at $300^{\circ} \mathrm{C}$ and $350^{\circ} \mathrm{C}$, respectively. (c) $I-V$ characteristics of sample B. Black, red, blue and green show the measured $V_{\mathrm{nl}}$ before annealing after annealing at $300{ }^{\circ} \mathrm{C}, 350^{\circ} \mathrm{C}$ and $400{ }^{\circ} \mathrm{C}$, respectively. The inset shows the $R A$ product normalized by the value before annealing measured at $1.0 \mathrm{~mA}$.

employed for the analyses. The spin diffusion length of the Si channel $\lambda$ was estimated from the curve fitting by using the following equation ${ }^{13}$ :

$$
V_{\mathrm{nl}}^{\mathrm{P}}\left(B_{z}\right)-V_{\mathrm{nl}}^{\mathrm{AP}}\left(B_{z}\right)= \pm S_{0} \int_{0}^{\infty} \sqrt{\frac{1}{4 \pi D t}} \cos (\omega t) \exp \left(-\frac{t}{\tau}\right) \exp \left(-\frac{L_{\mathrm{ch}}^{2}}{4 D t}\right) d t,
$$

where $B_{z}$ is the magnetic flux density along the $z$-direction, $S_{0}$ is a constant value that determines the magnitude of the signal, $D$ is the diffusion constant, $\omega=g \mu_{\mathrm{B}} B_{z} / \hbar$ is the Larmor frequency, $g=2$ is the $g$ factor for the electrons, $\mu_{\mathrm{B}}$ is the Bohr magneton, $\hbar$ is the Dirac constant and $\tau$ is the spin life time. The fitting curves obtained using Eq. (1) reproduced the experimental results well, as shown in Fig. 6 b. $\lambda=\sqrt{D \tau}$ was $1.6 \pm 0.1 \mu \mathrm{m}$ before annealing, $1.7 \pm 0.1 \mu \mathrm{m}$ after annealing at $300^{\circ} \mathrm{C}$, and $1.8 \pm 0.1 \mu \mathrm{m}$ after annealing at $350^{\circ} \mathrm{C}$. No significant changes in spin diffusion length were confirmed after annealing below $350^{\circ} \mathrm{C}$, as expected. Because the magnitude of the spin accumulation voltage was considerably small, the precise estimation of $\lambda$ after annealing at $400{ }^{\circ} \mathrm{C}$ was difficult. In fact, the interfacial resistance obviously decreased after annealing at $400{ }^{\circ} \mathrm{C}$ (see Fig. 6c), and the FM electrode degraded. However, identical characteristics were observed before annealing and after annealing at $300^{\circ} \mathrm{C}$ and $350^{\circ} \mathrm{C}$. The inset shows the resistance area products $R A$ normalized by the value before annealing. $R A$ slightly decreased after annealing at $350^{\circ} \mathrm{C}$ and steeply decreased to 0.85 after annealing at $400{ }^{\circ} \mathrm{C}$, which is similar trend as observed in the previous study ${ }^{16}$. Because nonlinear $I-V$ characteristics are maintained, the condition of the $\mathrm{MgO}$ barrier is expected to be different from that of sample $\mathrm{A}$, which might reflect an inherent thermal tolerance of the $\mathrm{Fe} / \mathrm{Co} / \mathrm{MgO}$ contact.

The thermal tolerance of $\mathrm{Mg}$ above the $\mathrm{MgO}$ structures is still an open question. The thermal tolerance of $\mathrm{NM}$ electrodes is largely improved, which allows for a direct comparison of the thermal tolerance of FM electrodes by the insertion of $\mathrm{Mg}$ below/above the $\mathrm{MgO}$ layer. Two samples, $\mathrm{C}$ and $\mathrm{D}$, with thermally tolerant $\mathrm{NM}$ electrodes and FM electrodes with a thin $\mathrm{Mg}$ layer below/above the MgO layer, were prepared; the FM electrodes of samples $\mathrm{C}$ and $\mathrm{D}$ consist of $\mathrm{Fe} / \mathrm{Co} / \mathrm{MgO} / \mathrm{Mg}$ and $\mathrm{Fe} / \mathrm{Co} / \mathrm{Mg} / \mathrm{MgO}$, respectively. The annealing temperatures of samples $\mathrm{C}$ and D were $320^{\circ} \mathrm{C}$ and $300^{\circ} \mathrm{C}$, respectively. Although a clear spin accumulation signal was obtained by L3T measurements even after annealing at $400^{\circ} \mathrm{C}$ for sample B, as shown in the inset of Fig. $6 \mathrm{a}$, the spin accumulation signals were strongly suppressed for sample C (Fig. 7a) and not detected for sample D (Fig. 7c) after annealing. The $I-V$ characteristics of their ferromagnetic electrodes are shown in Fig. $7 \mathrm{~b}$, d. The resistance decreased after annealing, which was consistent with the results observed in samples A and B. More importantly, the resistance decreased at much lower temperatures. Because the thermal tolerance of the NM electrodes is higher than $400^{\circ} \mathrm{C}$, the decrease in the thermal tolerance of the device is attributable to the $\mathrm{FM}$ electrodes including $\mathrm{Mg}$ layer and $0.8 \mathrm{~nm}$-thick MgO tunnelling barrier. The thermal tolerance more than $425^{\circ} \mathrm{C}$ was reported in $\mathrm{Co}_{2} \mathrm{FeSi} / \mathrm{MgO} /$ $\mathrm{Mg} / \mathrm{Si}$ structure employing $1.3 \mathrm{~nm}$-thick $\mathrm{MgO}$ tunnelling barrier ${ }^{16,31}$, indicating that the thick $\mathrm{MgO}$ provides better tolerance. However, we point out that large electrical resistance of the device due to the thick $\mathrm{MgO}$ hinders high magnetoresistance ratio. Although some improvements in spin polarization can be reported, the thermal tolerance worsens with the insertion of a Mg layer both below and above the MgO. 
a

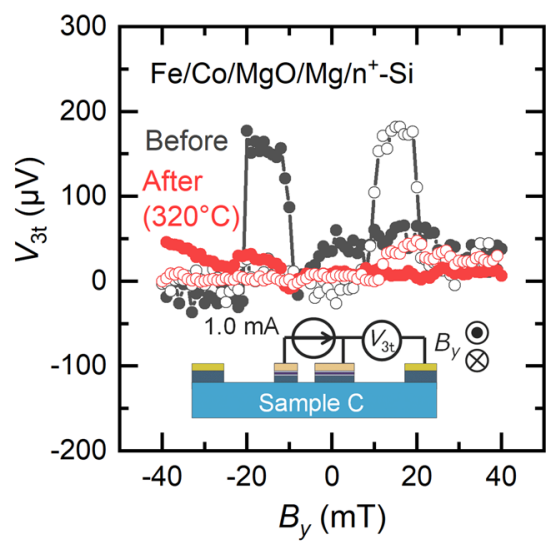

C

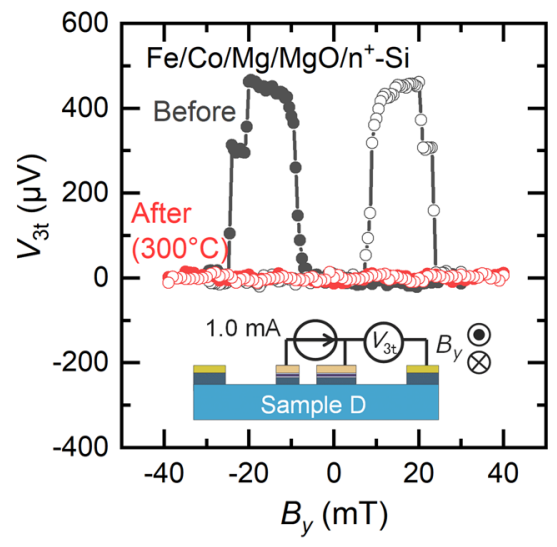

b

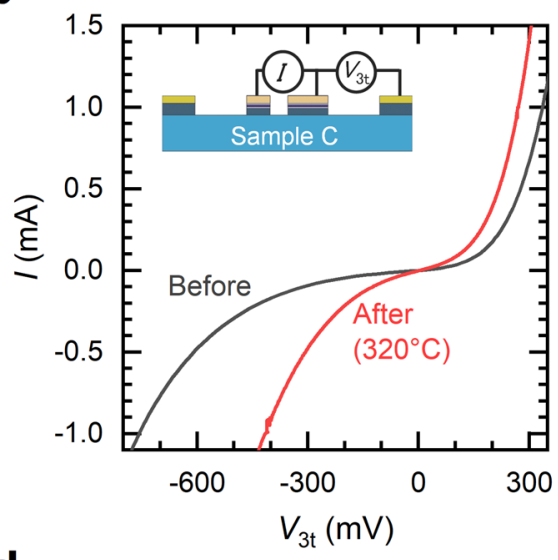

d

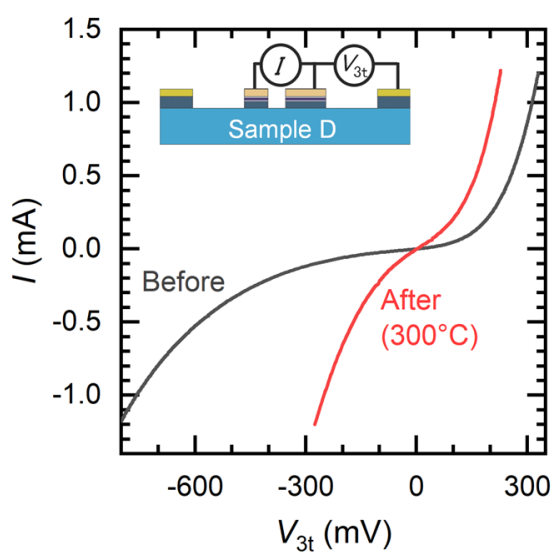

Figure 7. Results of the L3T measurements and $I-V$ measurements of samples $C$ and D. $L_{\mathrm{ch}}=2.75 \mu \mathrm{m}$, $t=100 \mathrm{~nm}$ for both samples. The widths of F1 and F2 were 0.5 and $2.0 \mu \mathrm{m}$, respectively. (a) Results of the L3T measurements of sample C. Black and red plots show the results before and after annealing, respectively. (b) Results of the $I-V$ measurements of sample C. Black and red plots show the results before and after annealing, respectively. (c) Results of the L3T measurements of sample D. Black and red plots show the results before and after annealing, respectively. (d) Results of the $I-V$ measurements of sample D. Black and red plots show the results before and after annealing, respectively.

\section{Conclusion}

In conclusion, we investigated the degradation mechanism of nondegenerate Si-based LSVs by thermal annealing and found two different mechanisms: Au diffusion from the NM electrode to the FM electrode and degradation of the $\mathrm{Mg}$ layer employed for FM electrodes. In the sample using $\mathrm{Au} / \mathrm{Ta} / \mathrm{Al}$ electrodes, interdiffusion of $\mathrm{Au}$ and $\mathrm{Al}$ atoms occurred, followed by the melting of $\mathrm{Au}$ and $\mathrm{Si}$ alloy at approximately $350{ }^{\circ} \mathrm{C}$ due to the eutectic alloy properties. The Au-Si liquid phase mainly diffused through the AlN/Si interface and finally broke the FM electrodes. By changing the structure from $\mathrm{Au} / \mathrm{Ta} / \mathrm{Al}$ to $\mathrm{Au} / \mathrm{Ta}$ with a 40-nm-thick Ta layer, the thermal tolerance was improved, and clear spin signals were obtained even after annealing at $400{ }^{\circ} \mathrm{C}$. The insertion of a thin $\mathrm{Mg}$ layer below or above the $\mathrm{MgO}$ layer was also examined, and their thermal tolerance was less than that without the $\mathrm{Mg}$ layer. The maximum thermal tolerance of $400^{\circ} \mathrm{C}$, which was sufficiently high for the post fabrication process of electronic devices was provided by the combination of $\mathrm{Au} / \mathrm{Ta}$ for $\mathrm{NM}$ and $\mathrm{Fe} / \mathrm{Co} / \mathrm{MgO}$ for $\mathrm{FM}$ electrodes. The thermally tolerant Si-based LSV certified the greater compatibility of Si-based spin devices with the electronics industry.

\section{Method}

Sample preparation. Four types of Si-based lateral spin valve devices were prepared. The spin valve structures were fabricated on a silicon-on-insulator substrate with a 100 -nm-thick $\mathrm{Si}(100)$ layer/200-nm-thick $\mathrm{SiO}_{2}$ layer/625- $\mu \mathrm{m}$-thick $\mathrm{Si}(100)$ substrate. Phosphorus $(\mathrm{P})$ atoms were doped into the Si layer by the ion implantation technique to form the $\mathrm{n}-\mathrm{Si}$ and $\mathrm{n}^{+}-\mathrm{Si}$ (only for sample A) layers. Then, rapid thermal annealing was carried out for their activation. The dopant concentration in the Si channel was confirmed by secondary-ion mass spectrometry, which showed a small distribution perpendicular to the plane in the range of $1 \times 10^{17}$ to $2 \times 10^{18} \mathrm{~cm}^{-3}$, indicating nondegenerate $\mathrm{Si}$. The dopant concentration of the 20 -nm-thick $\mathrm{n}^{+}$-Si layer employed to suppress the 
width of the depletion layer at the ferromagnetic contacts was $5 \times 10^{19} \mathrm{~cm}^{-3}$. The ferromagnetic contacts $\mathrm{F} 1$ and F2 were fabricated by electron beam deposition in an ultrahigh vacuum. Figure 1a shows the layer structures of these electrodes. The deposited materials were Fe $(12.4 \mathrm{~nm}) / \mathrm{CoFe}(0.6 \mathrm{~nm}) / \mathrm{MgO}(0.8 \mathrm{~nm})$ for sample A, Fe $(12.4 \mathrm{~nm}) / \mathrm{Co}(0.6 \mathrm{~nm}) / \mathrm{MgO}(0.8 \mathrm{~nm}) / \mathrm{n}^{+}-\mathrm{Si}(20 \mathrm{~nm}$ by sputtering $)$ for sample B, Fe $(12.4 \mathrm{~nm}) / \mathrm{Co}(0.6 \mathrm{~nm}) / \mathrm{MgO}$ $(0.8 \mathrm{~nm}) / \mathrm{Mg}(0.6 \mathrm{~nm}) / \mathrm{n}^{+}-\mathrm{Si}(20 \mathrm{~nm}$ by sputtering) for sample C and Fe $(12.4 \mathrm{~nm}) / \mathrm{Co}(0.6 \mathrm{~nm}) / \mathrm{Mg}(0.6 \mathrm{~nm}) /$ $\mathrm{MgO}(0.8 \mathrm{~nm}) / \mathrm{n}^{+}-\mathrm{Si}(20 \mathrm{~nm}$ by sputtering) for sample D. Electron beam lithography and Ar ion milling were employed to fabricate F1 and F2 and to remove the $\mathrm{n}^{+}$-Si layer in the channel region. AlN was deposited on the Si channel as a capping layer. Finally, the NM electrodes were fabricated using electron beam lithography and ion beam deposition. The NM materials of sample A were Au $(150 \mathrm{~nm}) / \mathrm{Ta}(5 \mathrm{~nm}) / \mathrm{Al}(40 \mathrm{~nm})$, while Au $(150 \mathrm{~nm}) /$ $\mathrm{Ta}(40 \mathrm{~nm})$ was employed for the other samples.

Thermal annealing. The temperature was increased to the set value within $30 \mathrm{~min}$, kept for $60 \mathrm{~min}$ and decreased naturally for $300 \mathrm{~min}$ using a Futek K-A102HP furnace. All the annealing processes were conducted in a vacuum $\left(\sim 1 \times 10^{-4} \mathrm{~Pa}\right)$.

Non-local four terminal (NL4T) method. A schematic of the current-voltage configuration is shown in the insets of Figs. $1 \mathrm{~b}$ and $5 \mathrm{a}$. A direct current was applied from N2 to F2. Voltage was measured at F1 and N1 under the magnetic field swept along the $y$-axis in Fig. 1a. Spin polarization $\beta$ was estimated by applying the onedimensional spin drift-diffusion equation ${ }^{32,33}$ to spin signal $\Delta V_{\mathrm{nl}}$. In this model, $\Delta V_{\mathrm{nl}}$ is expressed as:

$$
\Delta V_{\mathrm{nl}}=\frac{\beta^{2} J}{\left\{G_{\mathrm{N}}+\frac{1}{2}\left(G_{\mathrm{i} 1}+G_{\mathrm{i} 2}\right)+\frac{G_{\mathrm{i} 1} G_{\mathrm{i} 2}}{4 G_{\mathrm{N}}}\right\} \exp \left(\frac{L_{\mathrm{ch}}}{\lambda_{\mathrm{Si}}}\right)-\frac{G_{\mathrm{i} 1} G_{\mathrm{i} 2}}{4} \exp \left(-\frac{L_{\mathrm{ch}}}{\lambda_{\mathrm{Si}}}\right)},
$$

where $J$ is the charge current density, $\lambda_{\mathrm{Si}}$ is the spin diffusion length of $\mathrm{Si}, \sigma$ is the conductivity of $\mathrm{Si}, R A_{1}$ and $R A_{2}$ are the resistance area products of $\mathrm{F} 1$ and $\mathrm{F} 2, G_{\mathrm{N}}=\frac{\sigma}{\lambda_{\mathrm{Si}}}$ is the spin conductance of $\mathrm{Si}$ and $G_{\mathrm{i} 1(2)}=\frac{1}{R A_{1(2)}}$ is the spin conductance of the tunnel barrier of F1(F2). We assumed identical $\beta$ values for F1 and F2 and negligible spin resistance of the ferromagnetic metal. The experimentally measured values of $R A_{1}, R A_{2}$, and $\sigma$ were used for the analysis.

Local three terminal (L3T) method. A schematic of the current-voltage configuration is shown in the insets of Fig. 6a, c. A direct current of $1.0 \mathrm{~mA}$ was applied from F2 to F1. The external magnetic field was swept along the $y$-axis, and the voltage difference was measured at F2 and N2.

\section{Data availability}

The data that support the findings of this study are available from the corresponding author upon reasonable request.

Received: 21 December 2020; Accepted: 5 May 2021

Published online: 19 May 2021

\section{References}

1. Elliott, R. J. Theory of the effect of spin-orbit coupling on magnetic resonance in some semiconductors. Phys. Rev. 96, 266-279 (1954).

2. Huang, B., Monsma, D. J. \& Appelbaum, I. Coherent spin transport through a 350 micron thick silicon wafer. Phys. Rev. Lett. 99, 177209 (2007).

3. Lee, S. et al. Investigation of gating effect in Si spin MOSFET. Appl. Phys. Lett. 116, 022403 (2020).

4. Sasaki, T. et al. Spin transport in nondegenerate Si with a spin MOSFET structure at room temperature. Phys. Rev. Appl. 2, 034005 (2014).

5. Sugahara, S. \& Tanaka, M. A spin metal-oxide-semiconductor field-effect transistor using half-metallic-ferromagnet contacts for the source and drain. Appl. Phys. Lett. 84, 2307 (2004).

6. Matsuno, T., Sugahara, S. \& Tanaka, M. Novel reconfigurable logic gates using spin metal-oxide-semiconductor field-effect transistors. Jpn. J. Appl. Phys. 43, 6032-6037 (2004).

7. Tahara, T. et al. Room-temperature operation of Si spin MOSFET with high on/off spin signal ratio. Appl. Phys. Exp. 8, 113004 (2015).

8. Sato, S., Tanaka, M. \& Nakane, R. Spin transport in Si-based spin metal-oxide-semiconductor field-effect transistors: spin drift effect in the inversion channel and spin relaxation in the $\mathrm{n}^{+}$-Si source / drain regions. Phys. Rev. B 102, 035305 (2020).

9. Sato, S., Ichihara, M., Tanaka, M. \& Nakane, R. Electron spin and momentum lifetimes in two-dimensional Si accumulation channels: demonstration of Schottky-barrier spin metal-oxide-semiconductor field-effect transistors at room temperature. Phys. Rev. B 99, 165301 (2019).

10. Dery, H., Dalal, P., Cywiński, L. \& Sham, L. J. Spin-based logic in semiconductors for reconfigurable large-scale circuits. Nature 447, 573-576 (2007)

11. Ishihara, R. et al. Gate-tunable spin xor operation in a silicon-based device at room temperature. Phys. Rev. Appl. 13, 044010 (2020).

12. Lee, S. et al. Investigation of spin scattering mechanism in silicon channels of $\mathrm{Fe} / \mathrm{MgO} / \mathrm{Si}$ lateral spin valves. Appl. Phys. Lett. 110, 192401 (2017).

13. Sasaki, T. et al. Temperature dependence of spin diffusion length in silicon by Hanle-type spin precession. Appl. Phys. Lett. 96, $122101(2010)$

14. Ishikawa, M., Sugiyama, H., Inokuchi, T., Hamaya, K. \& Saito, Y. Spin transport and accumulation in n +-Si using Heusler compound $\mathrm{Co}_{2} \mathrm{FeSi} / \mathrm{MgO}$ tunnel contacts. Appl. Phys. Lett. 107, 092402 (2015).

15. Koike, T., Oogane, M., Tsunoda, M. \& Ando, Y. Large spin signals in $\mathrm{n}^{+}-\mathrm{Si} / \mathrm{MgO} / \mathrm{Co}_{2} \mathrm{Fe}_{0.4} \mathrm{Mn}_{0.6} \mathrm{Si}$ lateral spin-valve devices. J. Appl. Phys. 127, 085306 (2020).

16. Tiwari, A. et al. Effect of post annealing on spin accumulation and transport signals in $\mathrm{Co}_{2} \mathrm{FeSi} / \mathrm{MgO} / \mathrm{n}^{+}-\mathrm{Si}$ on insulator devices. AIP Adv. 6, 075119 (2016). 
17. Nakane, R., Ichihara, M., Sato, S. \& Tanaka, M. Nearly ideal spin tunneling efficiency in Fe/Mg/MgO/Si Ox/ $\mathrm{n}^{+}-\mathrm{Si}(001)$ junctions. Phys. Rev. Mater. 3, 024411 (2019).

18. Sato, S., Nakane, R., Hada, T. \& Tanaka, M. Spin injection into silicon in three-terminal vertical and four-terminal lateral devices with $\mathrm{Fe} / \mathrm{Mg} / \mathrm{MgO} / \mathrm{Si}$ tunnel junctions having an ultrathin $\mathrm{Mg}$ insertion layer. Phys. Rev. B 96, 235204 (2017).

19. Ishikawa, M. et al. Maximum magnitude in bias-dependent spin accumulation signals of $\mathrm{CoFe} / \mathrm{MgO} / \mathrm{Si}$ on insulator devices. J. Appl. Phys. 114, 243904 (2013).

20. Sugiyama, H. et al. Large spin-accumulation signal in Si for epitaxial CoFe/highly (100)-textured MgO/Si devices. Solid State Commun. 190, 49-52 (2014).

21. Yamashita, N. et al. Enhancement of spin signals by thermal annealing in silicon-based lateral spin valves enhancement of spin signals by thermal annealing in silicon-based lateral spin valves. AIP Adv. 10, 095021 (2020).

22. Cheng, Y.-L. et al. Effect of copper barrier dielectric deposition process on characterization of copper interconnect. J. Vac. Sci. Technol. B, Nanotechnol. Microelectron. Mater. Process. Meas. Phenom. 28, 567-572 (2010).

23. Hu, C. K., Rosenberg, R. \& Lee, K. Y. Electromigration path in Cu thin-film lines. Appl. Phys. Lett. 74, 2945-2947 (1999).

24. Theil, J. A., Mirkarimi, L., Fountain, G., Gao, G. \& Katkar, R. Recent developments in fine pitch wafer-to-wafer hybrid bonding with copper interconnect. In 2019 Int. Wafer Lev. Packag. Conf. IWLPC 2019 6-11 (2019). https://doi.org/10.23919/IWLPC.2019. 8913862.

25. Jedema, F. J., Filip, A. T. \& van Wees, B. J. Electrical spin injection and accumulation at room temperature in an all-metal mesoscopic spin valve. Nature 410, 345-348 (2001).

26. Sasaki, T. et al. Local magnetoresistance in Fe/MgO/Si lateral spin valve at room temperature. Appl. Phys. Lett. 104, 052404 (2014).

27. Lee, S. et al. Quantitative and systematic analysis of bias dependence of spin accumulation voltage in a nondegenerate Si-based spin valve. Phys. Rev. B 99, 064408 (2019).

28. Minho, O. \& Kajihara, M. Kinetics of solid-state reactive diffusion between Au and Al. Mater. Trans. 52, 677-684 (2011).

29. Koike, H. et al. Over $1 \%$ magnetoresistance ratio at room temperature in non-degenerate silicon-based lateral spin valves. Appl. Phys. Exp. 13, 083002 (2020).

30. Holloway, K. \& Fryer, P. M. Tantalum as a diffusion barrier between copper and silicon. Appl. Phys. Lett. 57, 1736 (1990).

31. Tiwari, A. et al. Room temperature observation of high spin polarization in post annealed $\mathrm{Co}_{2} \mathrm{FeSi} / \mathrm{MgO} / \mathrm{n}^{+}$-Si on insulator devices. Jpn. J. Appl. Phys. 56, 04CD05 (2017).

32. Yu, Z. G. \& Flatté, M. E. Electric-field dependent spin diffusion and spin injection into semiconductors. Phys. Rev. B 66, 201202(R) (2002).

33. Yu, Z. G. \& Flatté, M. E. Spin diffusion and injection in semiconductor structures: electric field effects. Phys. Rev. B 66, 235302 (2002)

\section{Acknowledgements}

N.Y. acknowledges the support from the Japan Society of the Promotion of Science (JSPS) Research Fellow Program (Grant No. 20J22776). A part of this study was supported by a Grant-in-Aid for Scientific Research from the Ministry of Education, Culture, Sports, Science and Technology (MEXT) of Japan, Grant-in-Aid for Scientific Research (S) "Semiconductor Spincurrentronics" No. 16H06330 and Grant-in-Aid for Scientific Research (B) 19H02197. N. Y. and S. L. acknowledge the JSPS Research Fellowship. A part of this work was supported by Kyoto University Nano Technology Hub in "Nanotechnology Platform Project" sponsored by the Ministry of Education, Culture, Sports, Science and Technology (MEXT), Japan.

\section{Author contributions}

N.Y. conducted the experiments, analysed the results, and wrote the paper. S.L., R.O. and E.S. checked the analysis. H.K., Y.S., S.M., and M.G. fabricated the samples. Y.A. and M.S. conceived the experiments. All authors discussed and commented on the manuscript.

\section{Competing interests}

The authors declare no competing interests.

\section{Additional information}

Correspondence and requests for materials should be addressed to N.Y.

Reprints and permissions information is available at www.nature.com/reprints.

Publisher's note Springer Nature remains neutral with regard to jurisdictional claims in published maps and institutional affiliations.

Open Access This article is licensed under a Creative Commons Attribution 4.0 International License, which permits use, sharing, adaptation, distribution and reproduction in any medium or format, as long as you give appropriate credit to the original author(s) and the source, provide a link to the Creative Commons licence, and indicate if changes were made. The images or other third party material in this article are included in the article's Creative Commons licence, unless indicated otherwise in a credit line to the material. If material is not included in the article's Creative Commons licence and your intended use is not permitted by statutory regulation or exceeds the permitted use, you will need to obtain permission directly from the copyright holder. To view a copy of this licence, visit http://creativecommons.org/licenses/by/4.0/.

(C) The Author(s) 2021 